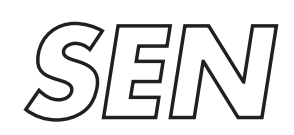

Software Engineering

$\int$ SEN $\begin{aligned} & \text { Software ENgineering } \\ & \text { On the axiomatizability of impossible futures: preorder } \\ & \text { versus equivalence }\end{aligned}$

T. Chen, W.J. Fokkink

Report SEN-R0801 MARCh 2008 
Centrum voor Wiskunde en Informatica (CWI) is the national research institute for Mathematics and Computer Science. It is sponsored by the Netherlands Organisation for Scientific Research (NWO).

CWI is a founding member of ERCIM, the European Research Consortium for Informatics and Mathematics.

CWI's research has a theme-oriented structure and is grouped into four clusters. Listed below are the names of the clusters and in parentheses their acronyms.

Probability, Networks and Algorithms (PNA)

\section{Software Engineering (SEN)}

Modelling, Analysis and Simulation (MAS)

Information Systems (INS)

Copyright (C) 2008, Stichting Centrum voor Wiskunde en Informatica

P.O. Box 94079, 1090 GB Amsterdam (NL)

Kruislaan 413, 1098 SJ Amsterdam (NL)

Telephone +31205929333

Telefax +31205924199

ISSN 1386-369X 


\title{
On the axiomatizability of impossible futures: preorder versus equivalence
}

\begin{abstract}
We investigate the (in)equational theory of impossible futures semantics over the process algebra BCCSP. We prove that no finite, sound axiomatization for BCCSP modulo impossible futures equivalence is ground-complete. By contrast, we present a finite, sound, groundcomplete axiomatization for BCCSP modulo impossible futures preorder\}. If the alphabet of actions is infinite, then this axiomatization is shown to be omega-complete. If the alphabet is finite, we prove that the inequational theory of BCCSP modulo impossible futures preorder lacks such a finite basis. We also derive non-finite axiomatizability results for nested impossible futures semantics.
\end{abstract}

2000 Mathematics Subject Classification: 68Q10, 68Q65, 68Q70

1998 ACM Computing Classification System: D.1.3, F.1.1, F.1.2

Keywords and Phrases: Concurrency; Process Algebra; 



\title{
On the Axiomatizability of Impossible Futures: Preorder versus Equivalence
}

\author{
Taolue Chen \\ $C W I$ \\ PO Box 94079, 1090 GB Amsterdam, NL \\ chen@cwi.nl \\ Wan Fokkink \\ Vrije Universiteit \\ De Boelelaan 1081a, 1081 HV Amsterdam, NL \\ wanf@cs.vu.nl
}

This work is partially supported by the Dutch Bsik project BRICKS.

\begin{abstract}
We investigate the (in)equational theory of impossible futures semantics over the process algebra BCCSP. We prove that no finite, sound axiomatization for BCCSP modulo impossible futures equivalence is groundcomplete. By contrast, we present a finite, sound, ground-complete axiomatization for BCCSP modulo impossible futures preorder. If the alphabet of actions is infinite, then this axiomatization is shown to be $\omega$-complete. If the alphabet is finite, we prove that the inequational theory of BCCSP modulo impossible futures preorder lacks such a finite basis. We also derive non-finite axiomatizability results for nested impossible futures semantics.
\end{abstract}

Keywords and Phrases: Concurrency, Process Algebra, Impossible Future Semantics, Axiomatizability, Ground-Completeness, $\omega$-completeness.

\section{Introduction}

Labeled transition systems constitute a widely used model of concurrent computation. They model processes by explicitly describing their states and their transitions from state to state, together with the actions that produce these transitions. Several notions of behavioral semantics have been proposed, with the aim to identify those states that afford the same observations. Van Glabbeek [6] presented the linear time - branching time spectrum of behavioral semantics for finitely branching, concrete, sequential processes. These semantics are based on simulation notions or on decorated traces. Fig. 1 depicts the linear time - branching time spectrum, where an arrow from one semantics to another means that the source of the arrow is finer, i.e. more discriminating, than the target.

In this paper, we study impossible futures semantics [11, 12]. This semantics is missing in van Glabbeek's original spectrum, because it was only studied seriously from $2001 \mathrm{on}$, the year that [6] appeared. An impossible future of a state $s$ consists of (1) a trace $s \stackrel{a_{1} \cdots a_{n}}{\rightarrow} s^{\prime}$, and (2) a set $X$ of traces such that $s^{\prime}$ does not exhibit any of the traces in $X$. Impossible futures semantics is a natural variant of possible futures semantics [10] (in which $X$ is the set of traces from $s^{\prime}$ ). In [8] it was shown that weak impossible futures equivalence (which takes into account the hidden action $\tau$ ) with an additional root condition, is the coarsest congruence with respect to choice and parallel composition operators containing weak bisimilarity with explicit divergence that respects deadlock/livelock traces and assigns unique solutions to recursive equations. This equivalence is closely related to fair testing semantics [9].

The process algebra BCCSP contains only the basic process algebraic operators from CCS and CSP, but is sufficiently powerful to express all finite synchronization trees (without $\tau$-transitions). Van 


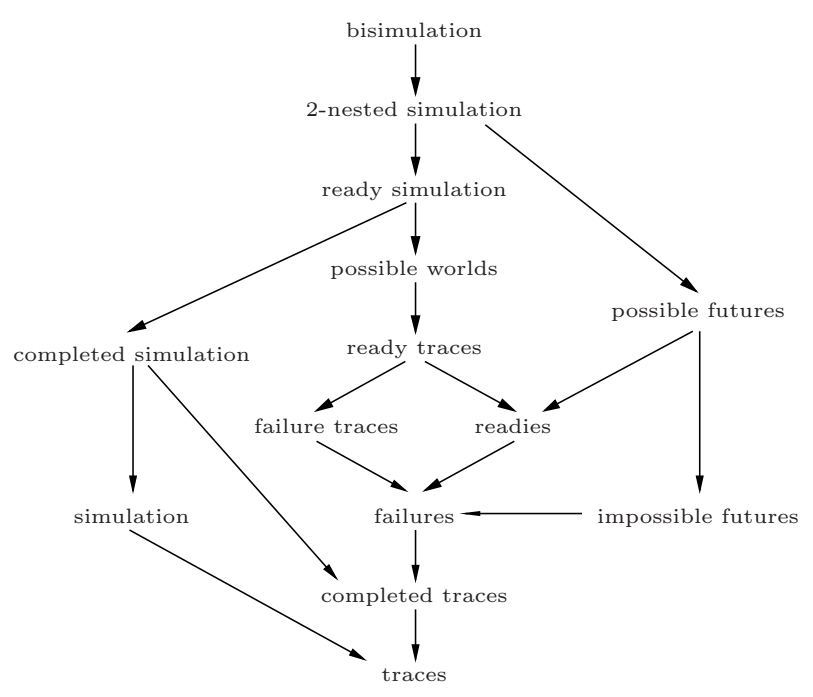

Figure 1. Linear time-branching time spectrum

Glabbeek [6] associated with most behavioral equivalences in his spectrum a sound axiomatization, to equate closed BCCSP terms that are behaviorally equivalent. These axiomatizations were shown to be ground-complete, meaning that whenever two closed BCCSP terms are behaviorally equivalent, then they can be equated.

An axiomatization is said to be $\omega$-complete if it enjoys the property that whenever all closed instances of an equation can be derived from it, then the equation itself can also be derived from it. In universal algebra, such an axiomatization is referred to as a basis for the equational theory of the algebra it axiomatizes. Groote [7] developed a technique of "inverted substitutions" to prove that an axiomatization is $\omega$-complete, and proved for some of the equivalences in the linear time - branching time spectrum that their equational theory in BCCSP has a finite basis. In [3, 4], a categorization of the equational theories for BCCSP modulo the semantics in the linear time - branching time spectrum is given. For each preorder and equivalence it is studied whether a finite, sound, ground-complete axiomatization exists. And if so, whether there exists a finite basis for the equational theory.

So all questions on these matters have been resolved? No, as for impossible futures semantics, the (in)equational theory remained unexplored. Only the inequational theory of BCCSP modulo weak impossible futures preorder was studied in [12]. In that paper, Voorhoeve and Mauw offer a finite, sound, ground-complete axiomatization; their ground-completeness proof relies heavily on the presence of $\tau$. They also prove that their axiomatization is $\omega$-complete (they do not refer to $\omega$-completeness explicitly, but they work on open terms, see [12, Thm. 5]). They implicitly assume an infinite alphabet (at $[12$, p. 7$]$ they require a different action for each variable).

In this paper, we focus on the axiomatizability of impossible futures preorder and equivalence over BCCSP. In summary, we obtain the following results.

1. We prove that there exists a finite, sound, ground-complete axiomatization for BCCSP modulo impossible futures preorder $\precsim_{\mathrm{IF}}{ }^{1}$. (By contrast, in [1] it was shown that such an axiomatization does not exist modulo possible futures preorder.)

2. Next, we show that BCCSP modulo impossible futures equivalence $\simeq_{\mathrm{IF}}$ does not have a finite, sound, ground-complete axiomatization. This negative result is based on the following infinite

\footnotetext{
${ }^{1}$ In case of an infinite alphabet of actions, occurrences of action names in axioms should be interpreted as variables, as else most of the axiomtizations would be infinite.
} 
family of equations from [1], for $m \geq 0$ :

$$
a^{2 m+1} \mathbf{0}+a\left(a^{m} \mathbf{0}+a^{2 m} \mathbf{0}\right) \approx a\left(a^{m} \mathbf{0}+a^{2 m} \mathbf{0}\right)
$$

Actually, since these equations are also sound modulo 2-nested simulation equivalence [6], this negative result applies to all BCCSP-congruences that are at least as fine as $\simeq_{\mathrm{IF}}$ and at least as coarse as 2-nested simulation equivalence.

3. Next, we investigate $\omega$-completeness for $\precsim$ IF .

First, we prove that if the alphabet of actions is infinite, then the ground-complete axiomatization for BCCSP modulo $\precsim_{I F}$ is $\omega$-complete. To prove this result, we apply the technique of inverted substitutions from [7]. Only, that technique was originally developed for equivalences. Therefore, as an aside, we adapt this technique in such a way that it applies to preorders.

Second, we prove that in case of a finite alphabet of actions, the inequational theory of BCCSP

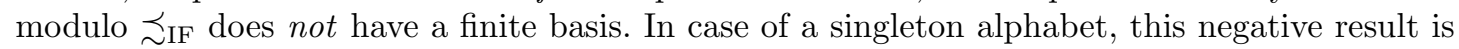
based on the following infinite family of equations, for $m \geq 0$ :

$$
a^{m} x \preccurlyeq a^{m} x+x
$$

And for finite alphabets with at least two actions, we use the family

$$
a\left(a^{m} x\right)+a\left(a^{m} x+x\right)+\sum_{b \in A} a\left(a^{m} x+a^{m} b \mathbf{0}\right) \preccurlyeq a\left(a^{m} x+x\right)+\sum_{b \in A} a\left(a^{m} x+a^{m} b \mathbf{0}\right)
$$

4. $n$-Nested impossible futures semantics, for $n \geq 0$, form a natural hierarchy (cf. [1]), which coincides with the universal relation for $n=0$, trace semantics for $n=1$, and impossible futures semantics for $n=2$. Using a proof strategy from [1], we show that the negative result regarding impossible futures equivalence extends to all $n$-nested impossible futures equivalences for $n \geq 2$, and to all $n$-nested impossible futures preorders for $n \geq 3$. Apparently, (2-nested) impossible futures preorder is the only positive exception.

To achieve the negative results, we mainly use what in [3, Sect. 2.3] is called the proof-theoretic technique. On top of this, a saturation principle is introduced, to transform a single summand into a large collection of (semi-)saturated summands.

Impossible futures semantics is the first example that affords a ground-complete axiomatization for BCCSP modulo the preorder, while missing a ground-complete axiomatization for BCCSP modulo the equivalence. This surprising fact suggests that if one wants to show $p \simeq_{\mathrm{IF}} q$, one has to resort to deriving $p \precsim_{\mathrm{IF}} q$ and $q \precsim_{\mathrm{IF}} p$ separately, instead of proving it directly.

In $[2,5]$ an algorithm is presented which produces, from an axiomatization for BCCSP modulo a preorder, an axiomatization for BCCSP modulo the corresponding equivalence. If the original axiomatization for the preorder is ground-complete or $\omega$-complete, then so is the resulting axiomatization for the equivalence. However, that algorithm only applies to semantics that are at least as coarse as ready simulation semantics. Since impossible futures semantics is incomparable to ready simulation semantics, it falls outside the scope of $[2,5]$. Interestingly, our results yield that no such algorithm exists for semantic incomparable with (or finer than) ready simulation.

This paper is set up as follows. Sect. 2 presents basic definitions regarding impossible futures semantics, the process algebra BCCSP, and (in)equational logic. Sect. 3 provides some basic facts for

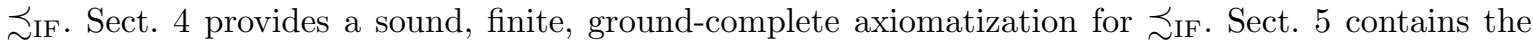
proof of the negative result for $\simeq_{\mathrm{IF}}$. Sect. 6 is devoted to the proofs of the negative and positive results regarding $\omega$-completeness for $\precsim$ IF. Sect. 7 contains the negative results regarding $n$-nested impossible futures semantics. 


\section{Preliminaries}

A labeled transition system consists of a set of states $S$, with typical element $s$, and a transition relation $\rightarrow \subseteq S \times L \times S$, where $L$ is a set of labels ranged over by $a, b$. We write $s \stackrel{a}{\rightarrow} s^{\prime}$ if $\left(s, a, s^{\prime}\right)$ is an element of $\rightarrow$. The set $\mathcal{I}(s)$ consists of those labels $a$ for which there exists an $s^{\prime}$ such that $s \stackrel{a}{\rightarrow} s^{\prime}$. Let $a_{1} \cdots a_{k}$, with $k \geq 0$, be a sequence of labels; we write $s \stackrel{a_{1} \cdots a_{k}}{\rightarrow} s^{\prime}$ if there are states $s_{0}, \ldots, s_{k}$ such that $s=s_{0} \stackrel{a_{1}}{\rightarrow} \cdots \stackrel{a_{k}}{\rightarrow} s_{k}=s^{\prime}$. A sequence $a_{1} \cdots a_{k}$ is a trace of a state $s$ if there is a state $s^{\prime}$ such that $s \stackrel{a_{1} \cdots a_{k}}{\rightarrow} s^{\prime}$. We write $\mathcal{T}(s)$ for the set of traces of state $s$, ranged over by $\alpha, \beta$. We say $a_{1} \cdots a_{k}$ is a completed trace of $s$ if moreover $\mathcal{I}\left(s^{\prime}\right)=\emptyset$, and write $\mathcal{C} \mathcal{T}(s)$ for the set of completed traces of state $s$. The empty sequence is denoted by $\varepsilon$. We write $s_{1} \precsim \mathrm{CT} s_{2}$ if the completed traces of $s_{1}$ are included in those of $s_{2}$.

Definition 1 Assume a labeled transition system. A pair $\left(a_{1} \cdots a_{k}, X\right)$, with $k \geq 0$ and $X \subseteq L^{*}$, is an impossible future of a state $s$ if $s \stackrel{a_{1} \cdots a_{k}}{\rightarrow} s^{\prime}$ for some state $s^{\prime}$ with $\mathcal{T}\left(s^{\prime}\right) \cap X=\emptyset$.

We write $s_{1} \precsim_{\mathrm{IF}} s_{2}$ if the impossible futures of $s_{1}$ are included in those of $s_{2}$. We write $s_{1} \simeq_{\mathrm{IF}} s_{2}$ if both $s_{1} \precsim_{\mathrm{IF}} s_{2}$ and $s_{2} \precsim_{\mathrm{IF}} s_{1}$. The relation $\precsim_{\mathrm{IF}}$ is called impossible futures preorder, while $\simeq_{\mathrm{IF}}$ is called impossible futures equivalence.

A sequence $a_{1} s_{1} \cdots a_{k} s_{k}$ is a completed path of a state $s_{0}$ if $s_{0} \stackrel{a_{1}}{\rightarrow} s_{1} \cdots \stackrel{a_{k}}{\rightarrow} s_{k}$ with $\mathcal{I}\left(s_{k}\right)=\emptyset$. We write $\mathcal{C P}(s)$ for the set of completed paths of state $s$, which is ranged over by $\pi$.

\subsection{BCCSP}

$\operatorname{BCCSP}(A)$ is a basic process algebra for expressing finite process behavior. Its signature consists of the constant $\mathbf{0}$, the binary operator ${ }_{-}+_{-}$, and unary prefix operators $a_{-}$, where $a$ ranges over a nonempty set $A$ of actions, called the alphabet, with typical elements $a, b$. The term $a^{n} t$ is obtained from $t$ by prefixing it $n$ times with $a$, i.e., $a^{0} t=t$ and $a^{n+1} t=a\left(a^{n} t\right)$. Intuitively, $\operatorname{closed} \operatorname{BCCSP}(A)$ terms, which are ranged over by $p, q, r$, represent finite process behaviors, where $\mathbf{0}$ does not exhibit any behavior, $p+q$ offers a choice between the behaviors of $p$ and $q$, and $a p$ executes action $a$ to transform into $p$. This intuition is captured by the transition rules below, in which $a$ ranges over $A$. They give rise to $A$-labeled transitions between closed BCCSP terms.

$$
\overline{a x \stackrel{a}{\rightarrow} x} \quad \frac{x \stackrel{a}{\rightarrow} x^{\prime}}{x+y \stackrel{a}{\rightarrow} x^{\prime}} \quad \frac{y \stackrel{a}{\rightarrow} y^{\prime}}{x+y \stackrel{a}{\rightarrow} y^{\prime}}
$$

We assume a countably infinite set $V$ of variables; $x, y, z$ denote elements of $V$. Open BCCSP terms, denoted by $t, u, v, w$, may contain variables from $V$. The set of variables that occur in term $t$ is denoted by $\operatorname{var}(t)$. And if $t \stackrel{a_{1} \cdots a_{k}}{\rightarrow} x+t^{\prime}$, for some $k \geq 0$, then $x \in \operatorname{var}_{k}(t)$. It is technically convenient to extend the operational semantics to open terms. We do not include additional rules for variables, which effectively means that they do not exhibit any behavior.

The depth of a term $t$, denoted by depth $(t)$, is the length of a longest trace of $t$. And the norm of a term $t$, denoted by norm $(t)$, is the length of a shortest completed trace of $t$.

A (closed) substitution, denoted by $\rho, \sigma$, maps variables in $V$ to (closed) terms. For open terms $t$ and $u$, and a preorder $\precsim$ (or equivalence $\simeq$ ) on closed terms, we define $t \precsim u$ (or $t \simeq u$ ) if $\rho(t) \precsim \rho(u$ ) (resp. $\rho(t) \simeq \rho(u))$ for all closed substitutions $\rho$. Clearly, $t \stackrel{a}{\rightarrow} t^{\prime}$ implies that $\sigma(t) \stackrel{a}{\rightarrow} \sigma\left(t^{\prime}\right)$ for all substitutions $\sigma$.

The preorders $\precsim$ in the linear time - branching time spectrum are all precongruences for BCCSP, meaning that $p_{1} \precsim q_{1}$ and $p_{2} \precsim q_{2}$ implies $p_{1}+p_{2} \precsim q_{1}+q_{2}$ and $a p_{1} \precsim a q_{1}$ for $a \in A$. (For (rooted weak) impossible futures preorder, a proof of this fact can be found in [12, Thm. 3].) And the equivalences in the spectrum are all congruences for BCCSP.

An axiomatization is a collection of equations $t \approx u$ or of inequations $t \preccurlyeq u$. The (in)equations in an axiomatization $E$ are referred to as axioms. If $E$ is an equational axiomatization, we write $E \vdash t \approx u$ 
if the equation $t \approx u$ is derivable from the axioms in $E$ using the rules of equational logic (reflexivity, symmetry, transitivity, substitution, and closure under BCCSP contexts). For the derivation of an inequation $t \preccurlyeq u$ from an inequational axiomatization $E$, denoted by $E \vdash t \preccurlyeq u$, the rule for symmetry is omitted. We will also allow equations $t \approx u$ in inequational axiomatizations, as an abbreviation of $t \preccurlyeq u$ and $u \preccurlyeq t$.

An axiomatization $E$ is sound modulo a preorder $\precsim$ (or equivalence $\simeq$ ) if for any terms $t, u$, from $E \vdash t \preccurlyeq u($ or $E \vdash t \approx u$ ) it follows that $\rho(t) \precsim \rho(u)$ (or $\rho(t) \simeq \rho(u)$ ) for all closed substitutions $\rho$. $E$ is ground-complete for $\precsim$ (or $\simeq$ ) if for any closed terms $p, q, p \precsim q$ (or $p \simeq q$ ) implies $E \vdash p \preccurlyeq q$ (or $E \vdash p \approx q$ ). And $E$ is $\omega$-complete if for any terms $t, u$ with $E \vdash \rho(t) \preccurlyeq \rho(u)$ (or $E \vdash \rho(t) \approx \rho(u)$ ) for all closed substitutions $\rho$, we have $E \vdash t \preccurlyeq u$ (or $E \vdash t \approx u$ ). The equational theory of BCCSP modulo a preorder $\precsim$ (or equivalence $\simeq$ ) is said to be finitely based if there exists a finite, $\omega$-complete axiomatization that is sound and ground-complete for BCCSP modulo $\precsim$ (or $\simeq$ ).

The core axioms A1-4 for BCCSP below are sound modulo every semantics in the spectrum depicted in Fig. 1. We assume that A1-4 are included in every axiomatization, and write $t=u$ if A1-4 $\vdash \approx u$.

$\begin{array}{rrl}\text { A1 } & x+y & \approx y+x \\ \text { A2 } & (x+y)+z & \approx x+(y+z) \\ \text { A3 } & x+x & \approx x \\ \text { A4 } & x+0 & \approx x\end{array}$

Summation $\sum\left\{t_{1}, \ldots, t_{n}\right\}$ or $\sum_{i \in\{1, \ldots, n\}} t_{i}$ denotes $t_{1}+\cdots+t_{n}$, where summation over the empty set denotes $\mathbf{0}$. As binding convention,,$_{-}+_{-}$and summation bind weaker than $a_{-}$. For every term $t$ there exists a finite set $\left\{a_{i} t_{i} \mid i \in I\right\}$ of terms and a finite set $Y$ of variables such that $t=\sum_{i \in I} a_{i} t_{i}+\sum_{y \in Y} y$. The $a_{i} t_{i}$ for $i \in I$ and the $y \in Y$ are called the summands of $t$ (notation: $a_{i} t_{i} \sqsubseteq t$ and $y \sqsubseteq t$ ). It is easy to see that $t \stackrel{a}{\rightarrow} t^{\prime}$ iff $a t^{\prime} \sqsubseteq t$.

\section{Properties of $\precsim$ IF}

We present some basic facts for $\precsim$ IF .

Lemma 1 Suppose $t \precsim$ IF $u$. Then

1. $\mathcal{T}(t)=\mathcal{T}(u)$; and

2. $\mathcal{C} \mathcal{T}(t) \subseteq \mathcal{C} \mathcal{T}(u)$.

Proof: Consider the closed substitution $\rho$ defined by $\rho(x)=\mathbf{0}$ for any $x \in V$. It is easy to see that for any term $v$, trace $\alpha$ and closed term $p$ :

$(*)$ if $\rho(v) \stackrel{\alpha}{\rightarrow} p$, then there is a term $v^{\prime}$ such that $v \stackrel{\alpha}{\rightarrow} v^{\prime}$ and $p=\rho\left(v^{\prime}\right)$.

1. Let $\alpha \in \mathcal{T}(t)$, i.e., $t \stackrel{\alpha}{\rightarrow} t^{\prime}$ for some $t^{\prime}$. Then $\rho(t) \stackrel{\alpha}{\rightarrow} \rho\left(t^{\prime}\right)$, so $(\alpha, \emptyset)$ is an impossible future of $\rho(t)$. Since $t \precsim$ IF $u,(\alpha, \emptyset)$ is also an impossible future of $\rho(u)$. So $\rho(u) \stackrel{\alpha}{\rightarrow} q$ for some $q$. By $(*), u \stackrel{\alpha}{\rightarrow} u^{\prime}$ for some $u^{\prime}$, and thus $\alpha \in \mathcal{T}(u)$. Hence $\mathcal{T}(t) \subseteq \mathcal{T}(u)$.

Let $\beta \in \mathcal{T}(u)$, i.e., $u \stackrel{\beta}{\rightarrow} u^{\prime}$ for some $u^{\prime}$. Then $\rho(u) \stackrel{\beta}{\rightarrow} \rho\left(u^{\prime}\right)$, so $(\varepsilon,\{\beta\})$ is not an impossible future of $\rho(u)$. Since $t \precsim$ IF $u,(\varepsilon,\{\beta\})$ is not an impossible future of $\rho(t)$ either. So $\rho(t) \stackrel{\beta}{\rightarrow} p$ for some $p$. By $(*), t \stackrel{\beta}{\rightarrow} t^{\prime}$ for some $t^{\prime}$, and thus $\beta \in \mathcal{T}(t)$. Hence $\mathcal{T}(u) \subseteq \mathcal{T}(t)$.

2. Let $\alpha \in \mathcal{C} \mathcal{T}(t)$, i.e., $t \stackrel{\alpha}{\rightarrow} \sum_{x \in X} x$ for some $X \subseteq V$. Then $\rho(t) \stackrel{\alpha}{\rightarrow} \mathbf{0}$, so $(\alpha, A)$ is an impossible future of $\rho(t)$. Since $t \precsim \operatorname{IF} u,(\alpha, A)$ is also an impossible future of $\rho(u)$. So $\rho(u) \stackrel{\alpha}{\rightarrow} \mathbf{0}$. By $(*)$, $u \stackrel{\alpha}{\rightarrow} u^{\prime}$ for some $u^{\prime}$ with $\rho\left(u^{\prime}\right)=\mathbf{0}$. So $u^{\prime}=\sum_{y \in Y} y$ for some $Y \subseteq V$, and thus $\alpha \in \mathcal{C T}(u)$. Hence $\mathcal{C} \mathcal{T}(t) \subseteq \mathcal{C} \mathcal{T}(u)$. 
Lemma 2 Suppose $t \precsim_{\mathrm{CT}} u$. Then $\operatorname{var}_{k}(t) \subseteq \operatorname{var}_{k}(u)$ for all $k \geq 0$.

Proof: Let $x \in \operatorname{var}_{k}(t)$, so that $t \stackrel{a_{1} \cdots a_{k}}{\longrightarrow} x+t^{\prime}$. Fix a $d>\operatorname{depth}(u)$, and consider the closed substitution $\rho$ defined by $\rho(x)=a^{d} \mathbf{0}$ and $\rho(y)=\mathbf{0}$ for any $y \neq x$. Then $\rho(t) \stackrel{a_{1} \cdots a_{k+d}}{\rightarrow} \mathbf{0}$ (with $a_{k+1} \cdots a_{k+d}=a^{d}$ ). Since $\rho(t) \precsim_{\text {CT }} \rho(u)$, it follows that $\rho(u) \stackrel{a_{1} \cdots a_{k+d}}{\longrightarrow}$. Since $d>\operatorname{depth}(u)$, clearly $u \stackrel{a_{1} \cdots a_{\ell}}{\rightarrow} z+u^{\prime}$ where $\rho(z) \stackrel{a_{\ell+1} \cdots a_{k+d}}{\rightarrow} \mathbf{0}$. Since $\ell \leq \operatorname{depth}(u)<d, \rho(z) \neq \mathbf{0}$, and hence $z=x$ and $\ell=k$. Concluding, $u \stackrel{a_{1} \cdots a_{k}}{\rightarrow} x+u^{\prime}$, so $x \in \operatorname{var}_{k}(u)$.

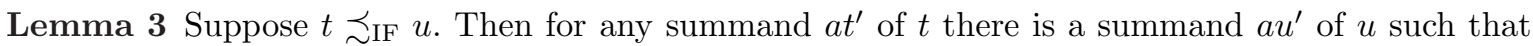
$\operatorname{var}\left(u^{\prime}\right) \subseteq \operatorname{var}\left(t^{\prime}\right)$.

Proof: Let $a t^{\prime}$ be a summand of $t$. Fix some $m>\operatorname{depth}(t)$, and consider the closed substitution $\rho$ defined by $\rho(x)=\mathbf{0}$ if $x \in \operatorname{var}\left(t^{\prime}\right)$ and $\rho(x)=a^{m} \mathbf{0}$ if $x \notin \operatorname{var}\left(t^{\prime}\right)$. Since $\rho(t) \stackrel{a}{\rightarrow} \rho\left(t^{\prime}\right)$ with $\operatorname{depth}\left(\rho\left(t^{\prime}\right)\right)=\operatorname{depth}\left(t^{\prime}\right)<m-1$, and $\rho(t) \precsim$ IF $\rho(u)$, clearly $\rho(u) \stackrel{a}{\rightarrow} q$ for some $q$ with $\operatorname{depth}(q)<m-1$. From the definition of $\rho$ it is then easy to see that $u$ must have a summand $a u^{\prime}$ with $\operatorname{var}\left(u^{\prime}\right) \subseteq \operatorname{var}\left(t^{\prime}\right)$.

Lemma 4 Let $|A|>1$. Suppose $t \precsim$ IF $u$. Then for any summand $a t^{\prime}$ of $t$ there is a summand $a u^{\prime}$ of $u$ such that $\operatorname{var}_{k}\left(u^{\prime}\right) \subseteq \operatorname{var}_{k}\left(t^{\prime}\right)$ for all $k \geq 0$.

Proof: Let $a t^{\prime}$ be a summand of $t$. Fix some $m>\operatorname{depth}(u)$, distinct actions $a, b \in A$, and an injection $\ulcorner\urcorner:. V \rightarrow \mathbb{Z}_{>0}$ (which exists because $V$ is countable). The closed substitution $\rho$ is defined by $\rho(z)=$ $a^{\ulcorner z\urcorner \cdot m} b \mathbf{0}$ for all $z \in V$. Since $\rho(t) \stackrel{a}{\rightarrow} \rho\left(t^{\prime}\right)$ and $\rho(t) \precsim$ IF $\rho(u)$, we have $\rho(u) \stackrel{a}{\rightarrow} q$ for some $q$ with $\mathcal{T}(q) \subseteq \mathcal{T}\left(\rho\left(t^{\prime}\right)\right)$.

$u$ cannot have a variable summand $x$ with $\sigma(x) \stackrel{a}{\rightarrow} q$. Namely, if this were the case, $q=a^{\ulcorner x\urcorner \cdot m-1} b \mathbf{0}$; but since $\ulcorner x\urcorner \cdot m-1 \geq \operatorname{depth}(t)>\operatorname{depth}\left(t^{\prime}\right)$, it follows from the definition of $\rho$ that $a\ulcorner x\urcorner \cdot m-1 b \notin \mathcal{T}\left(\rho\left(t^{\prime}\right)\right)$, contradicting $\mathcal{T}(q) \subseteq \mathcal{T}\left(\rho\left(t^{\prime}\right)\right)$.

Hence, there must be a summand $a u^{\prime}$ of $u$ with $\rho\left(u^{\prime}\right)=q$. Let $y \in \operatorname{var}_{k}\left(u^{\prime}\right)$ for some $k \geq 0$. That is, $u^{\prime} \stackrel{a_{1} \cdots a_{k}}{\longrightarrow} y+u^{\prime \prime}$, so $a_{1} \cdots a_{k} a^{\ulcorner}{ }^{\urcorner\urcorner \cdot m} b \in \mathcal{T}\left(\rho\left(u^{\prime}\right)\right) \subseteq \mathcal{T}\left(\rho\left(t^{\prime}\right)\right)$. Since $k \leq \operatorname{depth}\left(u^{\prime}\right)<m$, it then follows from the definition of $\rho$ that $t^{\prime}{\stackrel{a_{1} \cdots a_{k}}{\longrightarrow}}^{\rightarrow} y+t^{\prime \prime}$ for some $t^{\prime \prime}$. So $y \in \operatorname{var}_{k}\left(t^{\prime}\right)$. Concluding, $\operatorname{var}_{k}\left(u^{\prime}\right) \subseteq \operatorname{var}_{k}\left(t^{\prime}\right)$ for all $k \geq 0$.

Remark: The condition $|A|>1$ in Lem. 4 is necessary. Namely, if $|A|=1$, then for instance aax $\precsim_{\text {IF }}$ $a(a x+x)$.

\section{Axiomatization for $\precsim_{\mathrm{IF}}$}

In this section, we provide a ground-complete axiomatization for impossible futures preorder. It consists of the core axioms A1-4 together with two extra axioms:

$$
\begin{aligned}
& \text { IF1 } \quad a(x+y) \preccurlyeq a x+a y \\
& \text { IF2 } a(x+y)+a x+a(y+z) \approx a x+a(y+z)
\end{aligned}
$$

Recall that here, $t \approx u$ denotes that both $t \preccurlyeq u$ and $u \preccurlyeq t$ are present in the inequational axiomatization. It is not hard to see that IF 1,2 are sound modulo $\precsim_{\text {IF }}$. The rest of this section is devoted to proving the following theorem.

Theorem 1 A1-4+IF1-2 is ground-complete for $\operatorname{BCCSP}(A)$ modulo $\precsim$ IF.

To give some intuition on the ground-completeness proof, we first present an example. 
Example 1 Let $p=a\left(a \mathbf{0}+a^{2} \mathbf{0}\right)+a^{4} \mathbf{0}$ and $q=a\left(a \mathbf{0}+a^{3} \mathbf{0}\right)+a^{3} \mathbf{0}$. It is not hard to see that $p \precsim$ IF $q$. However, neither $a\left(a \mathbf{0}+a^{2} \mathbf{0}\right) \precsim_{\text {IF }} a\left(a \mathbf{0}+a^{3} \mathbf{0}\right)$ nor $a\left(a \mathbf{0}+a^{2} \mathbf{0}\right) \precsim$ IF $a^{3} \mathbf{0}$ holds. In order to derive $p \preccurlyeq q$, we therefore first derive with IF2 that $q \approx p+q$. And $p \preccurlyeq p+q$ can be derived with IF1.

In general, to derive a sound closed inequation $p \preccurlyeq q$, first we derive $q \approx \mathbb{S}(q)$ (see Lem. 5 ), where $\mathbb{S}(q)$ contains for every $a \in \mathcal{I}(q)$ a "saturated" $a$-summand (see Def. 2). (In Ex. 1, this saturated $a$-summand would have the form $a\left(a \mathbf{0}+a^{2} \mathbf{0}+a^{3} \mathbf{0}+a\left(a \mathbf{0}+a^{2} \mathbf{0}\right)\right)$.) Then, in the proof of Thm. 1, we derive $\Psi+\mathbb{S}(q) \approx \mathbb{S}(q)$ (equation (4.1)), $p \preccurlyeq \Psi$ (equation (4.2)) and $p \preccurlyeq p+q$ (equation (4.3)), where the closed term $\Psi$ is built from many "semi-saturated" summands (like, in Ex. 1, $p$ ). These results together provide the desired proof (see the last line of the proof of Thm. 1).

Definition 2 For each closed term $q$, the closed term $\mathbb{S}(q)$ is defined recursively on the depth of $q$ as follows:

$$
\mathbb{S}(q)=q+\sum_{a \in \mathcal{I}(q)} a\left(\mathbb{S}\left(\sum_{a q^{\prime} \sqsubseteq q} q^{\prime}\right)\right)
$$

Example 2 If $q=a(b(c \mathbf{0}+d \mathbf{0})+b e \mathbf{0})+a f \mathbf{0}$, then $\mathbb{S}(q)=a(b(c \mathbf{0}+d \mathbf{0})+b e \mathbf{0})+a f \mathbf{0}+a(b(c \mathbf{0}+d \mathbf{0})+$ $b e \mathbf{0}+f \mathbf{0}+b(c \mathbf{0}+d \mathbf{0}+e \mathbf{0}))$.

In the remainder of this section, $E$ denotes the axiomatization A1-4+IF1-2.

Lemma 5 For each closed term $q, E \vdash q \approx \mathbb{S}(q)$.

Proof: By induction on $\operatorname{depth}(q)$. For any $a \in \mathcal{I}(q)$,

$$
E \vdash q \approx q+a\left(\sum_{a q^{\prime} \sqsubseteq q} q^{\prime}\right) \approx q+a\left(\mathbb{S}\left(\sum_{a q^{\prime} \sqsubseteq q} q^{\prime}\right)\right)
$$

The first derivation step uses IF2, and the second induction. Hence, summing up over all $a \in \mathcal{I}(q)$,

$$
E \vdash q \approx q+\sum_{a \in \mathcal{I}(q)} a\left(\mathbb{S}\left(\sum_{a q^{\prime} \sqsubseteq q} q^{\prime}\right)\right)=\mathbb{S}(q)
$$

For closed terms $q$ and $\alpha \in \mathcal{T}(q)$, the closed term $q_{\alpha}$ is obtained by summing over all closed terms $q^{\prime}$ such that $q \stackrel{\alpha}{\rightarrow} q^{\prime}$, and then applying the saturation from Def. 2 . The auxiliary terms $q_{\alpha}$ will only be used in the derivation of equation (4.1) within the proof of Thm. 1.

Definition 3 Given a closed term $q$, and a completed trace $a_{1} \cdots a_{d}$ of $q$. For $0 \leq \ell \leq d$ we define

$$
Q_{a_{1} \cdots a_{\ell}}=\left\{q_{\ell} \mid q \stackrel{a_{1}}{\rightarrow} q_{1} \cdots \stackrel{a_{\ell}}{\rightarrow} q_{\ell}\right\}
$$

and

$$
q_{a_{1} \cdots a_{\ell}}=\mathbb{S}\left(\sum_{q_{\ell} \in Q_{a_{1} \cdots a_{\ell}}} q_{\ell}\right)
$$

Note that $q_{\varepsilon}=\mathbb{S}(q)$. We prove some basic properties for the terms $q_{\alpha}$.

Lemma 6 Given a closed term $q$, and a completed trace $a_{1} \cdots a_{d}$ of $q$. Then, for $0 \leq \ell<d$,

- $q_{a_{1} \cdots a_{\ell}} \stackrel{a_{\ell+1}}{\rightarrow} q_{a_{1} \cdots a_{\ell+1}} ;$ and 
- $q_{a_{1} \cdots a_{\ell}} \stackrel{a_{\ell+1}}{\rightarrow} q_{\ell+1} \quad$ for all $q_{\ell+1} \in Q_{a_{1} \cdots a_{\ell+1}}$.

Proof: Clearly, $q_{\ell+1} \in Q_{a_{1} \cdots a_{\ell+1}}$ iff there exists some $q_{\ell} \in Q_{a_{1} \cdots a_{\ell}}$ such that $q_{\ell} \stackrel{a_{\ell+1}}{\rightarrow} q_{\ell+1}$. And since $a_{1} \cdots a_{\ell+1}$ is a trace of $q, a_{\ell+1} \in \mathcal{I}\left(q_{\ell}\right)$ for some $q_{\ell} \in Q_{a_{1} \cdots a_{\ell}}$. So by Def. 2,

$$
q_{a_{1} \cdots a_{\ell}}=\mathbb{S}\left(\sum_{q_{\ell} \in Q_{a_{1} \cdots a_{\ell}}} q_{\ell}\right) \stackrel{a_{\ell+1}}{\rightarrow} \mathbb{S}\left(\sum_{q_{\ell+1} \in Q_{a_{1} \cdots a_{\ell+1}}} q_{\ell+1}\right)=q_{a_{1} \cdots a_{\ell+1}}
$$

Moreover, for all $q_{\ell+1} \in Q_{a_{1} \cdots a_{\ell+1}}$ we have $\sum_{q_{\ell} \in Q_{a_{1} \cdots a_{\ell}}} q_{\ell} \stackrel{a_{\ell+1}}{\rightarrow} q_{\ell+1}$. Hence, by Def. 2,

$$
q_{a_{1} \cdots a_{\ell}}=\mathbb{S}\left(\sum_{q_{\ell} \in Q_{a_{1} \cdots a_{\ell}}} q_{\ell}\right) \stackrel{a_{\ell+1}}{\rightarrow} q_{\ell+1}
$$

We now embark on proving the promised ground-completeness result.

Proof: (of Thm. 1) Suppose $p \precsim$ IF $q$. We derive $E \vdash p \preccurlyeq q$ using induction on depth ( $p$ ). If $p=\mathbf{0}$, then clearly $q=\mathbf{0}$, and we are done. So assume $p \neq \mathbf{0}$, and consider any completed path $\pi=a_{1} p_{1} \cdots a_{d} p_{d}$ of $p$ (with $d \geq 1$ ); that is, $p \stackrel{a_{1}}{\rightarrow} p_{1} \cdots \stackrel{a_{d}}{\rightarrow} p_{d}=\mathbf{0}$. We recursively construct closed terms $\psi_{\ell}^{\pi}$, for $\ell$ from $d$ down to 1 . For the base case, $\psi_{d}^{\pi}=\mathbf{0}$. Now let $1 \leq \ell<d$. Since $p \stackrel{a_{1} \cdots a_{\ell}}{\rightarrow} p_{\ell}$ and $p \precsim$ IF $q$, there exists a sequence of transitions $q \stackrel{a_{1} \cdots a_{\ell}}{\rightarrow} q_{\ell}$ such that $\mathcal{T}\left(q_{\ell}\right) \subseteq \mathcal{T}\left(p_{\ell}\right)$. We define

$$
\psi_{\ell}^{\pi}=q_{\ell}+a_{\ell+1} \psi_{\ell+1}^{\pi}
$$

We prove, by induction on $d-\ell$, that for $1 \leq \ell \leq d$,

$$
\mathcal{T}\left(\psi_{\ell}^{\pi}\right) \subseteq \mathcal{T}\left(p_{\ell}\right)
$$

The base case is trivial, since $\mathcal{T}\left(\psi_{d}^{\pi}\right)=\emptyset$. Now let $1 \leq \ell<d$. By induction, $\mathcal{T}\left(\psi_{\ell+1}^{\pi}\right) \subseteq \mathcal{T}\left(p_{\ell+1}\right)$. Moreover, $p_{\ell} \stackrel{a_{\ell+1}}{\longrightarrow} p_{\ell+1}$, so $\mathcal{T}\left(a_{\ell+1} \psi_{\ell+1}^{\pi}\right) \subseteq \mathcal{T}\left(p_{\ell}\right)$. Hence, $\mathcal{T}\left(\psi_{\ell}^{\pi}\right)=\mathcal{T}\left(q_{\ell}+a_{\ell+1} \psi_{\ell+1}^{\pi}\right)=\mathcal{T}\left(q_{\ell}\right) \cup$ $\mathcal{T}\left(a_{\ell+1} \psi_{\ell+1}^{\pi}\right) \subseteq \mathcal{T}\left(p_{\ell}\right)$.

Next, we prove, by induction on $d-\ell$, that for $1 \leq \ell \leq d$,

$$
E \vdash a_{\ell} \psi_{\ell}^{\pi}+q_{a_{1} \cdots a_{\ell-1}} \approx q_{a_{1} \cdots a_{\ell-1}}
$$

In the base case, since $\psi_{d}^{\pi}=\mathbf{0} \in Q_{a_{1} \cdots a_{d}}$ (see Def. 3), this is a direct consequence of the second item in Lem. 6. Now let $1 \leq \ell<d$.

$$
\begin{aligned}
& E \vdash \quad a_{\ell} \psi_{\ell}^{\pi}+q_{a_{1} \cdots a_{\ell-1}} \\
& =a_{\ell}\left(q_{\ell}+a_{\ell+1} \psi_{\ell+1}^{\pi}\right)+q_{a_{1} \cdots a_{\ell-1}} \\
& +a_{\ell} q_{\ell}+a_{\ell} q_{a_{1} \cdots a \ell} \\
& \approx a_{\ell}\left(q_{\ell}+a_{\ell+1} \psi_{\ell+1}^{\pi}\right)+q_{a_{1} \cdots a_{\ell-1}} \\
& +a_{\ell} q_{\ell}+a_{\ell}\left(a_{\ell+1} \psi_{\ell+1}^{\pi}+q_{a_{1} \cdots a_{\ell}}\right) \quad \text { (induction) } \\
& \approx q_{a_{1} \cdots a_{\ell-1}}+a_{\ell} q_{\ell} \\
& +a_{\ell}\left(q_{a_{1} \cdots a_{\ell}}+a_{\ell+1} \psi_{\ell+1}^{\pi}\right) \\
& \approx q_{a_{1} \cdots a_{\ell-1}}+a_{\ell} q_{\ell} \\
& +a_{\ell} q_{a_{1} \cdots a_{\ell}} \\
& =q_{a_{1} \cdots a_{\ell-1}}
\end{aligned}
$$


In the end, for $\ell=1$, we get $E \vdash a_{1} \psi_{1}^{\pi}+q_{\varepsilon} \approx q_{\varepsilon}$. In other words,

$$
E \vdash a_{1} \psi_{1}^{\pi}+\mathbb{S}(q) \approx \mathbb{S}(q)
$$

Since this holds for all completed paths $\pi$ of $p$, it follows that

$$
E \vdash \sum_{a \in \mathcal{I}(p)} \sum_{a p^{\prime} \sqsubseteq p} \sum_{\pi \in \mathcal{C} \mathcal{P}\left(a p^{\prime}\right)} a \psi_{1}^{\pi}+\mathbb{S}(q) \approx \mathbb{S}(q)
$$

where $\mathcal{C P}\left(a p^{\prime}\right)$ denotes the set of completed paths of the summand $a p^{\prime}$.

On the other hand, for every summand $a p^{\prime}$ of $p$,

$$
p^{\prime} \precsim \sum_{\pi \in \mathcal{C P}\left(a p^{\prime}\right)} \psi_{1}^{\pi}
$$

Namely, consider any path $\pi_{0}=a_{1} p_{1} \cdots a_{h} p_{h}$ of $a p^{\prime}$. Extend $\pi_{0}$ to some completed path $\pi$ of $a p^{\prime}$. By the definition of the $\psi_{\ell}^{\pi}$, clearly, $\psi_{\ell}^{\pi} \stackrel{a_{\ell+1}}{\rightarrow} \psi_{\ell+1}^{\pi}$ for $1 \leq \ell<h$. So $\psi_{1}^{\pi} \stackrel{a_{2} \cdots a_{h}}{\rightarrow} \psi_{h}^{\pi}$. Moreover, we proved that $\mathcal{T}\left(\psi_{h}^{\pi}\right) \subseteq \mathcal{T}\left(p_{h}\right)$.

So by induction on depth, for every summand $a p^{\prime}$ of $p$,

$$
E \vdash p^{\prime} \preccurlyeq \sum_{\pi \in \mathcal{C} \mathcal{P}\left(a p^{\prime}\right)} \psi_{1}^{\pi}
$$

And thus, by IF1,

$$
E \vdash a p^{\prime} \preccurlyeq a\left(\sum_{\pi \in \mathcal{C} \mathcal{P}\left(a p^{\prime}\right)} \psi_{1}^{\pi}\right) \preccurlyeq \sum_{\pi \in \mathcal{C} \mathcal{P}\left(a p^{\prime}\right)} a \psi_{1}^{\pi}
$$

Hence, summing over all summands $a p^{\prime}$ of $p$,

$$
E \vdash p \preccurlyeq \sum_{a \in \mathcal{I}(p)} \sum_{a p^{\prime} \sqsubseteq p} \sum_{\pi \in \mathcal{C} \mathcal{P}\left(a p^{\prime}\right)} a \psi_{1}^{\pi}
$$

Finally, since $p \precsim_{\text {IF }} q$, clearly, for each $a \in \mathcal{I}(p)$,

$$
\sum_{a p^{\prime} \sqsubseteq p} p^{\prime} \precsim \mathrm{IF} \sum_{a q^{\prime} \sqsubseteq q} q^{\prime}
$$

So by induction on depth, for each $a \in \mathcal{I}(p)$,

$$
E \vdash \sum_{a p^{\prime} \sqsubseteq p} p^{\prime} \preccurlyeq \sum_{a q^{\prime} \sqsubseteq q} q^{\prime}
$$

So by IF2 and IF1, and since $\mathcal{I}(p)=\mathcal{I}(q)$,

$$
\begin{aligned}
E & \vdash p \approx p+\sum_{a \in \mathcal{I}(p)} a\left(\sum_{a p^{\prime} \sqsubseteq p} p^{\prime}\right) \\
& \preccurlyeq p+\sum_{a \in \mathcal{I}(q)} a\left(\sum_{a q^{\prime} \sqsubseteq q} q^{\prime}\right) \preccurlyeq p+\sum_{a \in \mathcal{I}(q)} \sum_{a q^{\prime} \sqsubseteq q} a q^{\prime}
\end{aligned}
$$

That is,

$$
E \vdash p \preccurlyeq p+q
$$

Finally, inequations (4.3), (4.2) and (4.1), together with Lem. 5, yield

$$
\begin{aligned}
& E \vdash p \preccurlyeq p+q \approx p+\mathbb{S}(q) \preccurlyeq \\
& \sum_{a \in \mathcal{I}(p)} \sum_{a p^{\prime} \sqsubseteq p} \sum_{\pi \in \mathcal{C} \mathcal{P}\left(a p^{\prime}\right)} a \psi_{1}^{\pi}+\mathbb{S}(q) \approx \mathbb{S}(q) \approx q
\end{aligned}
$$




\section{Non-finite Axiomatizability of $\simeq_{\mathrm{IF}}$}

In this section, we prove that surprisingly, there does not exist any finite, sound, ground-complete axiomatization for $\operatorname{BCCSP}(A)$ modulo $\simeq_{\mathrm{IF}}$. The cornerstone for this negative result is the following infinite family of closed equations, for $m \geq 0$ :

$$
a^{2 m+1} \mathbf{0}+a\left(a^{m} \mathbf{0}+a^{2 m} \mathbf{0}\right) \approx a\left(a^{m} \mathbf{0}+a^{2 m} \mathbf{0}\right)
$$

It is not hard to see that they are sound modulo $\simeq_{\mathrm{IF}}$. We start with a key lemma.

Lemma 7 Assume that, for some terms $t, u$ and closed substitution $\rho$ :

1. $t \precsim$ IF $u$;

2. $m>\operatorname{depth}(u)$;

3. $\mathcal{C} \mathcal{T}(\rho(u)) \subseteq\left\{a^{m+1}, a^{2 m+1}\right\}$; and

4. there is a closed term $p^{\prime}$ such that $\rho(t) \stackrel{a}{\rightarrow} p^{\prime}$ and $\mathcal{C} \mathcal{T}\left(p^{\prime}\right)=\left\{a^{2 m}\right\}$.

Then there is a closed term $q^{\prime}$ such that $\rho(u) \stackrel{a}{\rightarrow} q^{\prime}$ and $\mathcal{C} \mathcal{T}\left(q^{\prime}\right)=\left\{a^{2 m}\right\}$.

Proof: According to proviso (4) of the lemma, we can distinguish two cases.

- $t$ has a summand $y \in V$ such that $\rho(y) \stackrel{a}{\rightarrow} p^{\prime}$ where $\mathcal{C} \mathcal{T}\left(p^{\prime}\right)=\left\{a^{2 m}\right\}$. Since $t \precsim$ IF $u$, by Lem. 2 , $y$ is also a summand of $u$. Hence $\rho(u) \stackrel{a}{\rightarrow} p^{\prime}$.

- $t$ has a summand $a t^{\prime}$ with $\mathcal{C} \mathcal{T}\left(\rho\left(t^{\prime}\right)\right)=\left\{a^{2 m}\right\}$. Since depth $\left(t^{\prime}\right)<m$, clearly, either norm $(\rho(x))=0$ or $\operatorname{norm}(\rho(x))>m$ for any $x \in \operatorname{var}\left(t^{\prime}\right)$. Since $t \precsim$ IF $u$, by Lem. 3, $u$ has a summand $a u^{\prime}$ with $\operatorname{var}\left(u^{\prime}\right) \subseteq \operatorname{var}\left(t^{\prime}\right)$. Hence, either norm $(\rho(x))=0$ or norm $(\rho(x))>m$ for any $x \in \operatorname{var}\left(u^{\prime}\right)$. Since $\operatorname{depth}\left(u^{\prime}\right)<m, a^{m} \notin \mathcal{C} \mathcal{T}\left(\rho\left(u^{\prime}\right)\right)$. It follows from $\mathcal{C} \mathcal{T}(\rho(u)) \subseteq\left\{a^{m+1}, a^{2 m+1}\right\}$ that $\mathcal{C} \mathcal{T}\left(\rho\left(u^{\prime}\right)\right)=$ $\left\{a^{2 m}\right\}$.

Lemma 8 Let the finite axiomatization $E$ be sound modulo $\simeq_{I F}$. Assume that, for some closed terms $p, q$ :

1. $E \vdash p \approx q$;

2. $m>\max \{\operatorname{depth}(u) \mid t \approx u \in E\}$;

3. $\mathcal{C} \mathcal{T}(q) \subseteq\left\{a^{m+1}, a^{2 m+1}\right\} ;$ and

4. there is a closed term $p^{\prime}$ such that $p \stackrel{a}{\rightarrow} p^{\prime}$ and $\mathcal{C} \mathcal{T}\left(p^{\prime}\right)=\left\{a^{2 m}\right\}$.

Then there is a closed term $q^{\prime}$ such that $q \stackrel{a}{\rightarrow} q^{\prime}$ and $\mathcal{C} \mathcal{T}\left(q^{\prime}\right)=\left\{a^{2 m}\right\}$.

Proof: By induction on the derivation of $E \vdash p \approx q$.

- Case $E \vdash p \approx q$ because $\rho(t)=p$ and $\rho(u)=q$ for some $t \approx u \in E$ and closed substitution $\rho$. The claim follows by Lem. 7 .

- Case $E \vdash p \approx q$ because $E \vdash p \approx r$ and $E \vdash r \approx q$ for some $r$. By proviso (3) of the lemma and Lem. $1(2), \mathcal{C} \mathcal{T}(r) \subseteq\left\{a^{m+1}, a^{2 m+1}\right\}$. Since there is a $p^{\prime}$ such that $p \stackrel{a}{\rightarrow} p^{\prime}$ with $\mathcal{C} \mathcal{T}\left(p^{\prime}\right)=\left\{a^{2 m}\right\}$, by induction, there is an $r^{\prime}$ such that $r \stackrel{a}{\rightarrow} r^{\prime}$ and $\mathcal{C} \mathcal{T}\left(r^{\prime}\right)=\left\{a^{2 m}\right\}$. Hence, again by induction, there is a $q^{\prime}$ such that $q \stackrel{a}{\rightarrow} q^{\prime}, \mathcal{C} \mathcal{T}\left(q^{\prime}\right)=\left\{a^{2 m}\right\}$. 
- Case $E \vdash p \approx q$ because $p=p_{1}+p_{2}$ and $q=q_{1}+q_{2}$ with $E \vdash p_{1} \approx q_{1}$ and $E \vdash p_{2} \approx q_{2}$. Since there is a $p^{\prime}$ such that $p \stackrel{a}{\rightarrow} p^{\prime}$ and $\mathcal{C} \mathcal{T}\left(p^{\prime}\right)=\left\{a^{2 m}\right\}$, either $p_{1} \stackrel{a}{\rightarrow} p^{\prime}$ or $p_{2} \stackrel{a}{\rightarrow} p^{\prime}$. Assume, without loss of generality, that $p_{1} \stackrel{a}{\rightarrow} p^{\prime}$. By induction, there is a $q^{\prime}$ such that $q_{1} \stackrel{a}{\rightarrow} q^{\prime}$ and $\mathcal{C} \mathcal{T}\left(q^{\prime}\right)=\left\{a^{2 m}\right\}$. Since $q_{1} \neq \mathbf{0}$, clearly $\mathcal{C} \mathcal{T}\left(q_{1}\right) \subseteq\left\{a^{m+1}, a^{2 m+1}\right\}$. So $q=q_{1}+q_{2} \stackrel{a}{\rightarrow} q^{\prime}$.

- Case $E \vdash p \approx q$ because $p=a p^{\prime}$ and $q=a q^{\prime}$ with $E \vdash p^{\prime} \approx q^{\prime}$. By proviso $(4), \mathcal{C} \mathcal{T}\left(p^{\prime}\right)=\left\{a^{2 m}\right\}$. So by Lem. $1(2), \mathcal{C} \mathcal{T}\left(q^{\prime}\right)=\left\{a^{2 m}\right\}$.

Remark: Lem. 8 does not hold if its first requirement is changed into $E \vdash p \preccurlyeq q$. Note that the proof regarding the congruence rule for $a$.- in Lem. 8 fails for $\precsim$ IF .

For example, consider the following closed inequations, for $m \geq 0$ :

$$
a^{2 m+1} \mathbf{0} \preccurlyeq a\left(a^{2 m} \mathbf{0}+a^{m} \mathbf{0}\right)
$$

They are sound modulo $\precsim_{\mathrm{IF}}$, and satisfy the third and fourth requirement of Lem. 8. However, they can all be derived by means of IF1:

$$
\begin{aligned}
& a^{2 m+1} \mathbf{0}=a\left(a^{m}\left(a^{m}+\mathbf{0}\right)\right) \preccurlyeq a\left(a^{m-1}\left(a^{m+1} \mathbf{0}+a \mathbf{0}\right)\right) \\
& \preccurlyeq a\left(a^{m-2}\left(a^{m+2} \mathbf{0}+a^{2} \mathbf{0}\right)\right) \preccurlyeq \cdots \preccurlyeq a\left(a^{2 m} \mathbf{0}+a^{m} \mathbf{0}\right)
\end{aligned}
$$

Theorem 2 There is no finite, sound, ground-complete axiomatization for $\operatorname{BCCSP}(A)$ modulo $\simeq_{\mathrm{IF}}$.

Proof: Let $E$ be a finite axiomatization over $\operatorname{BCCSP}(A)$ that is sound modulo $\simeq_{\mathrm{IF}}$. Let $m$ be greater than the depth of any term in $E$. Clearly, $a\left(a^{m} \mathbf{0}+a^{2 m} \mathbf{0}\right)$ does not contain a summand $r$ such that $r \stackrel{a}{\rightarrow} r^{\prime}$ and $\mathcal{C} \mathcal{T}\left(r^{\prime}\right)=\left\{a^{2 m}\right\}$. So according to Lem. 8, $a^{2 m+1} \mathbf{0}+a\left(a^{m} \mathbf{0}+a^{2 m} \mathbf{0}\right) \approx a\left(a^{m} \mathbf{0}+a^{2 m} \mathbf{0}\right)$ cannot be derived from $E$. And this closed inequation is sound modulo $\simeq_{\mathrm{IF}}$.

Actually, since the equations $a^{2 m+1} \mathbf{0}+a\left(a^{m} \mathbf{0}+a^{2 m} \mathbf{0}\right) \approx a\left(a^{m} \mathbf{0}+a^{2 m} \mathbf{0}\right)$ are sound modulo 2-nested simulation equivalence, this negative result applies to all BCCSP-congruences that are at least as fine as impossible futures equivalence and at least as coarse as 2-nested simulation equivalence.

\section{6. $\omega$-Completeness for $\precsim$ IF}

In this section, we turn to $\omega$-completeness. In view of the negative result on impossible futures equivalence in Sect. 5, we focus on impossible futures preorder. In case $|A|=\infty$, we prove that there exists a finite basis for the equational theory of $\operatorname{BCCSP}(A)$ modulo $\precsim$ IF. The proof is based on an adaptation of Groote's inverted substitutions technique [7] to inequations. In case $|A|<\infty$, we prove that a finite basis does not exist. We give two different proofs of this last fact, one for the case $1<|A|<\infty$ and one for the case $|A|=1$.

6.1 $|A|=\infty$

The axiomatization A1-4+IF1-2 is $\omega$-complete, provided the alphabet is infinite. Our proof of this fact, which is omitted here, is based on inverted substitutions [7]; actually, while Groote developed this technique for equivalences, here we need it for preorders.

Let $\mathrm{T}(\Sigma)$ and $\mathbb{T}(\Sigma)$ denote the set of closed and open terms, respectively, over some signature $\Sigma$. Consider an axiomatization $E$ over $\Sigma$. For each inequation $t \preccurlyeq u$ of which all closed instances can be derived from $E$, one must define a closed substitution $\rho$ and a mapping $R: \mathrm{T}(\Sigma) \rightarrow \mathbb{T}(\Sigma)$ such that:

(1) $E \vdash t \preccurlyeq R(\rho(t))$ and $E \vdash R(\rho(u)) \preccurlyeq u$; 
(2) $E \vdash R(\sigma(v)) \preccurlyeq R(\sigma(w))$ for each $v \preccurlyeq w \in E$ and closed substitution $\sigma$; and

(3) for each function symbol $f$ (with arity $n$ ) in the signature, and for all closed terms $p_{1}, \ldots, p_{n}, q_{1}, \ldots, q_{n}$ :

$$
\begin{array}{r}
E \cup\left\{p_{i} \preccurlyeq q_{i}, R\left(p_{i}\right) \preccurlyeq R\left(q_{i}\right) \mid i=1, \ldots, n\right\} \vdash \\
R\left(f\left(p_{1}, \ldots, p_{n}\right)\right) \preccurlyeq R\left(f\left(q_{1}, \ldots, q_{n}\right)\right)
\end{array}
$$

Then, as proved in Appendix I, $E$ is $\omega$-complete.

Theorem 3 For $|A|=\infty$, A1-4+IF1-2 is $\omega$-complete.

Proof: Consider any pair of $\operatorname{BCCSP}(A)$ terms $t, u$. Define the closed substitution $\rho$ by $\rho(y)=a_{y} \mathbf{0}$, where $a_{y}$ is a unique action for $y \in V$ that occurs in neither $t$ nor $u$. Such actions exist because $A$ is infinite. We define the mapping $R$ from closed to open $\operatorname{BCCSP}(A)$ terms as follows:

$$
\begin{cases}R(\mathbf{0}) & =\mathbf{0} \\ R(a t) & =y \text { if } a=a_{y} \text { for some } y \in V \\ R(a t) & =a R(t) \text { if } a \neq a_{y} \text { for all } y \in V \\ R(t+u) & =R(t)+R(u)\end{cases}
$$

We check the three aforementioned properties.

(1) Since $t$ and $u$ do not contain actions of the form $a_{y}$, clearly $R(\rho(t))=t$ and $R(\rho(u))=u$.

(2) For A1-4, the proof is trivial. We check the remaining cases IF1 and IF2. Let $\sigma$ be a closed substitution. We first consider IF1. We distinguish two cases.

$-a=a_{y}$ for some $y \in V$. Then $R\left(a_{y}\left(\sigma\left(x_{1}\right)+\sigma\left(x_{2}\right)\right)\right)=y \preccurlyeq y+y=R\left(a_{y}\left(\sigma\left(x_{1}\right)\right)+a_{y}\left(\sigma\left(x_{2}\right)\right)\right)$.

$$
\begin{aligned}
& -a \neq a_{y} \text { for all } y \in V \text {. Then } R\left(a\left(\sigma\left(x_{1}\right)+\sigma\left(x_{2}\right)\right)\right)=a\left(R\left(\sigma\left(x_{1}\right)\right)+R\left(\sigma\left(x_{2}\right)\right)\right) \\
& \quad \preccurlyeq a R\left(\sigma\left(x_{1}\right)\right)+a R\left(\sigma\left(x_{2}\right)\right)=R\left(a \sigma\left(x_{1}\right)+a \sigma\left(x_{2}\right)\right) .
\end{aligned}
$$

We now turn to IF2. We distinguish two cases as well.

$$
\begin{aligned}
& -a=a_{y} \text { for some } y \in V \text {. Then } R\left(a_{y}\left(\sigma\left(x_{1}\right)+\sigma\left(x_{2}\right)\right)+a_{y} \sigma\left(x_{1}\right)+a_{y}\left(\sigma\left(x_{2}\right)+\sigma\left(x_{3}\right)\right)\right)= \\
& \quad y+y+y \approx y+y=R\left(a_{y} \sigma\left(x_{1}\right)+a_{y}\left(\sigma\left(x_{2}\right)+\sigma\left(x_{3}\right)\right)\right) . \\
& -a \neq a_{y} \text { for all } y \in V \text {. Then } R\left(a\left(\sigma\left(x_{1}\right)+\sigma\left(x_{2}\right)\right)+a \sigma\left(x_{1}\right)+a\left(\sigma\left(x_{2}\right)+\sigma\left(x_{3}\right)\right)\right)=a\left(R\left(\sigma\left(x_{1}\right)\right)+\right. \\
& \left.\quad R\left(\sigma\left(x_{2}\right)\right)\right)+a R\left(\sigma\left(x_{1}\right)\right)+a\left(R\left(\sigma\left(x_{2}\right)\right)+R\left(\sigma\left(x_{3}\right)\right)\right) \\
& \quad \approx a R\left(\sigma\left(x_{1}\right)\right)+a\left(R\left(\sigma\left(x_{2}\right)\right)+R\left(\sigma\left(x_{3}\right)\right)\right)=R\left(a \sigma\left(x_{1}\right)+a\left(\sigma\left(x_{2}\right)+\sigma\left(x_{3}\right)\right)\right) .
\end{aligned}
$$

(3) Consider the operator ${ }_{-}+_{-}$. From $R\left(p_{1}\right) \preccurlyeq R\left(q_{1}\right)$ and $R\left(p_{2}\right) \preccurlyeq R\left(q_{2}\right)$ we derive $R\left(p_{1}+p_{2}\right)=$ $R\left(p_{1}\right)+R\left(p_{2}\right) \preccurlyeq R\left(q_{1}\right)+R\left(q_{2}\right)=R\left(q_{1}+q_{2}\right)$.

Consider the prefix operator $a_{-}$. We distinguish two cases.

$-a=a_{y}$ for some $y \in V$. Then $R\left(a_{y} p_{1}\right)=y=R\left(a_{y} q_{1}\right)$.

$-a \neq a_{y}$ for all $y \in V$. Then from $R\left(p_{1}\right) \preccurlyeq R\left(q_{1}\right)$ we derive $R\left(a p_{1}\right)=a R\left(p_{1}\right) \preccurlyeq a R\left(q_{1}\right)=$ $R\left(a q_{1}\right)$. 
$6.21<|A|<\infty$

In this section, we prove that, if $A$ is finite, the inequational theory of $\operatorname{BCCSP}(A)$ modulo $\precsim$ IF does not have a finite basis. The cornerstone for this negative result is the following infinite family of inequations, for $m \geq 0$ :

$$
a\left(a^{m} x\right)+\Phi_{m} \preccurlyeq \Phi_{m}
$$

with

$$
\Phi_{m}=a\left(a^{m} x+x\right)+\sum_{b \in A} a\left(a^{m} x+a^{m} b \mathbf{0}\right)
$$

It is not hard to see that these inequations are sound modulo $\precsim$ IF. Namely, given a closed substitution $\rho, \mathcal{I}\left(\rho\left(a\left(a^{m} x\right)\right)\right)=\{a\}=\mathcal{I}\left(\rho\left(\Phi_{m}\right)\right)$. And if $\rho\left(a\left(a^{m} x\right)\right) \stackrel{a_{1} \cdots a_{k}}{\rightarrow} p$ with $k \geq 2$, then owing to the summand $a\left(a^{m} x+x\right)$, we have $\rho\left(\Phi_{m}\right) \stackrel{a_{1} \cdots a_{k}}{\rightarrow} p$. Finally, consider the transition $\rho\left(a\left(a^{m} x\right)\right) \stackrel{a}{\rightarrow} a^{m} \rho(x)$. If $\rho(x)=\mathbf{0}$, then clearly $\rho\left(\Phi_{m}\right) \stackrel{a}{\rightarrow} a^{m} \mathbf{0}$. And if $b \in \mathcal{I}(\rho(x))$ for some $b \in A$, then clearly $\rho\left(\Phi_{m}\right) \stackrel{a}{\rightarrow} a^{m} \rho(x)+a^{m} b \mathbf{0}$, and $\mathcal{T}\left(a^{m} \rho(x)+a^{m} b \mathbf{0}\right)=\mathcal{T}\left(a^{m} \rho(x)\right)$. Concluding, for any $\alpha$, if $\rho\left(a\left(a^{m} x\right)\right) \stackrel{\alpha}{\rightarrow} p$, then $\rho\left(\Phi_{m}\right) \stackrel{\alpha}{\rightarrow} q$ with $\mathcal{T}(q)=\mathcal{T}(p)$.

We now establish some key lemmas.

Lemma 9 Let $1<|A|<\infty$. Assume that, for some terms $t, u$ and substitution $\sigma$ :

1. $t \precsim \mathrm{IF} u$;

2. $m>\operatorname{depth}(u)$

3. $\sigma(u)+\Phi_{m} \simeq_{\mathrm{IF}} \Phi_{m} ;$ and

4. $\sigma(t)$ has a summand $\simeq_{\mathrm{IF}} a\left(a^{m} x\right)$.

Then $\sigma(u)$ has a summand $\simeq_{\mathrm{IF}} a\left(a^{m} x\right)$.

Proof: According to proviso (4) of the lemma, we can distinguish two cases.

- $t$ has a summand $y \in V$ such that $\sigma(y)$ has a summand $\simeq_{\mathrm{IF}} a\left(a^{m} x\right)$. Since $t \precsim$ IF $u$, by Lem. 2 , $y$ is also a summand of $u$. Hence $\sigma(u)$ has a summand $\simeq_{\mathrm{IF}} a\left(a^{m} x\right)$.

- $t$ has a summand $a t^{\prime}$ with $\sigma\left(t^{\prime}\right) \simeq_{\mathrm{IF}} a^{m} x$. Since $t \precsim \precsim_{\mathrm{IF}} u$ and $|A|>1$, by Lem. 4, there is a summand $a u^{\prime}$ of $u$ such that $\operatorname{var}_{k}\left(u^{\prime}\right) \subseteq \operatorname{var}_{k}\left(t^{\prime}\right)$ for all $k \geq 0$. Since $\sigma\left(t^{\prime}\right) \simeq_{\mathrm{IF}} a^{m} x$, by Lem. 1(1), depth $\left(\sigma\left(t^{\prime}\right)\right)=m$, so for all $k \geq 0$ and $z \in \operatorname{var}_{k}\left(u^{\prime}\right) \subseteq \operatorname{var}_{k}\left(t^{\prime}\right), \operatorname{depth}(\sigma(z)) \leq m-k$. Moreover, proviso $(2)$ implies $\operatorname{depth}\left(u^{\prime}\right)<m$, so it follows that $\operatorname{depth}\left(\sigma\left(u^{\prime}\right)\right) \leq m$. On the other hand, it follows from proviso (3) of the lemma together with Lem. 1(2) that norm $\left(\sigma\left(u^{\prime}\right)\right) \geq m$. So all completed traces of $\sigma\left(u^{\prime}\right)$ are of the form $\sigma\left(u^{\prime}\right) \stackrel{a^{m}}{\rightarrow} u^{\prime \prime}$.

Since $\sigma\left(t^{\prime}\right) \simeq_{\mathrm{IF}} a^{m} x$, by Lem. 2, $\operatorname{var}_{m}\left(\sigma\left(t^{\prime}\right)\right)=\{x\}$ and $\operatorname{var}_{k}\left(\sigma\left(t^{\prime}\right)\right)=\emptyset$ for $k \neq m$. Since $\operatorname{var}_{k}\left(u^{\prime}\right) \subseteq \operatorname{var}_{k}\left(t^{\prime}\right)$ for all $k \geq 0$, it follows that $\operatorname{var}_{m}\left(\sigma\left(u^{\prime}\right)\right) \subseteq\{x\}$ and $\operatorname{var}_{k}\left(\sigma\left(u^{\prime}\right)\right)=\emptyset$ for $k \neq m$. Due to proviso (3) of the lemma, it is easy to see that for each completed trace $\sigma\left(u^{\prime}\right) \stackrel{a^{m}}{\rightarrow} u^{\prime \prime}, u^{\prime \prime} \neq \mathbf{0}$; so $\operatorname{var}_{m}\left(\sigma\left(u^{\prime}\right)\right) \subseteq\{x\}$ yields $u^{\prime \prime}=x$. Concluding, $\sigma\left(u^{\prime}\right) \simeq_{\text {IF }} a^{m} x$.

Lemma 10 Let $1<|A|<\infty$. Assume that, for some terms $t, u$ :

1. $E \vdash t \preccurlyeq u$;

2. $m>\max \{\operatorname{depth}(w) \mid v \preccurlyeq w \in E\}$;

3. $u+\Phi_{m} \simeq_{\mathrm{IF}} \Phi_{m}$; and 
4. $t$ has a summand $\simeq_{\mathrm{IF}} a\left(a^{m} x\right)$.

Then $u$ has a summand $\simeq_{\mathrm{IF}} a\left(a^{m} x\right)$.

Proof: By induction on the derivation of $E \vdash p \preccurlyeq q$.

- Case $E \vdash t \preccurlyeq u$ because $\sigma(v)=t$ and $\sigma(w)=u$ for some $v \preccurlyeq w \in E$ and substitution $\sigma$. The claim follows by Lem. 9 .

- Case $E \vdash t \preccurlyeq u$ because $E \vdash t \preccurlyeq v$ and $E \vdash v \preccurlyeq u$ for some $v$. Since $v \precsim$ IF $u$ and $u+\Phi_{m} \simeq_{\mathrm{IF}} \Phi_{m}$, clearly $v+\Phi_{m} \simeq_{\mathrm{IF}} \Phi_{m}$. By induction, $v$ has a summand $\simeq_{\mathrm{IF}} a\left(a^{m} x\right)$. Hence, again by induction, $u$ has a summand $\simeq_{\mathrm{IF}} a\left(a^{m} x\right)$.

- Case $E \vdash t \preccurlyeq u$ because $t=t_{1}+t_{2}$ and $u=u_{1}+u_{2}$ with $E \vdash t_{1} \preccurlyeq u_{1}$ and $E \vdash t_{2} \preccurlyeq t_{2}$. Since $t$ has a summand $\simeq_{\mathrm{IF}} a\left(a^{m} x\right)$. so does either $t_{1}$ or $t_{2}$. Assume, without loss of generality, that $t_{1}$ does. Since $u+\Phi_{m} \simeq_{\mathrm{IF}} \Phi_{m}$, clearly $u_{1}+\Phi_{m} \simeq_{\mathrm{IF}} \Phi_{m}$. By induction, $u_{1}$ has a summand $\simeq_{\mathrm{IF}} a\left(a^{m} x\right)$, so the same holds for $u$.

- Case $E \vdash t \preccurlyeq u$ because $t=a t^{\prime}$ and $u=a u^{\prime}$ with $E \vdash t^{\prime} \preccurlyeq u^{\prime}$. By proviso (4) of the lemma, $t^{\prime} \simeq_{\mathrm{IF}} a^{m} x$. Hence $a^{m} x \precsim_{\mathrm{IF}} u^{\prime}$. So by Lem. 1(1), depth $\left(u^{\prime}\right)=m$. On the other hand, it follows from proviso (3) of the lemma together with Lem. 1(2) that norm $\left(u^{\prime}\right) \geq m$. So all completed traces of $u$ are of the form $u^{\prime} \stackrel{a^{m}}{\rightarrow} u^{\prime \prime}$.

Since $a^{m} x \precsim_{\text {IF }} u^{\prime}$ and $m>1$ and $|A|>1$, clearly, $\operatorname{var}_{0}\left(u^{\prime}\right)=\emptyset$. And from proviso (3) of the lemma together with Lem. 2 it follows that $\operatorname{var}_{m}\left(u^{\prime}\right) \subseteq\{x\}$ and $\operatorname{var}_{k}\left(u^{\prime}\right)=\emptyset$ for $k \notin\{0, m\}$. Due to proviso (3) of the lemma, it is easy to see that for each completed trace $u^{\prime} \stackrel{a^{m}}{\rightarrow} u^{\prime \prime}, u^{\prime \prime} \neq \mathbf{0}$; so $\operatorname{var}_{m}\left(u^{\prime}\right) \subseteq\{x\}$ yields $u^{\prime \prime}=x$. Concluding, $u^{\prime} \simeq_{\mathrm{IF}} a^{m} x$.

Theorem 4 For $1<|A|<\infty$, the inequational theory of $\operatorname{BCCSP}(A) \operatorname{modulo} \precsim_{\text {IF }}$ does not have a finite basis.

Proof: Let $E$ be a finite axiomatization over $\operatorname{BCCSP}(A)$ that is sound modulo $\precsim$ IF. Let $m$ be greater than the depth of any term in $E$. Clearly, $\Phi_{m}$ does not contain a summand $\simeq_{\mathrm{IF}} a\left(a^{m} x\right)$. So according to Lem. 10, $a\left(a^{m} x\right)+\Phi_{m} \preccurlyeq \Phi_{m}$ cannot be derived from $E$. And this inequation is sound modulo $\precsim$ IF.

6.3 $|A|=1$

In this section, we prove that the inequational theory of $\operatorname{BCCSP}(A)$ modulo $\precsim$ IF does not have a finite basis in case of a singleton alphabet. The cornerstone for the negative result for $|A|=1$ is the following infinite family of inequations, for $m \geq 0$ :

$$
a^{m} x \preccurlyeq a^{m} x+x
$$

If $|A|=1$, then these inequations are clearly sound modulo $\precsim$ IF. Note that given a closed substitution $\rho, \mathcal{T}(\rho(x)) \subseteq \mathcal{T}\left(\rho\left(a^{m} x\right)\right)$

Lemma 11 If $t \precsim_{\mathrm{IF}} a^{m} x+x$, then $t \simeq_{\mathrm{IF}} a^{m} x+x$ or $t \simeq_{\mathrm{IF}} a^{m} x$.

Proof: The case $m=0$ is trivial; we focus on the case $m>0$. Since $t \precsim$ IF $a^{m} x+x$, by Lem. 2, $\operatorname{var}_{0}(t) \subseteq\{x\}, \operatorname{var}_{m}(t) \subseteq\{x\}$, and $\operatorname{var}_{k}(t)=\emptyset$ for $k \notin\{0, m\}$. Furthermore, by Lem. $1(2), \mathcal{C} \mathcal{T}(t) \subseteq$ $\left\{a^{m}\right\}$, and clearly $t \stackrel{a^{m}}{\rightarrow} \mathbf{0}$; so all completed traces of $t$ are of the form $t \stackrel{a^{m}}{\rightarrow} x$. Hence, either $t \simeq_{\mathrm{IF}} a^{m} x+x$ or $t \simeq_{\mathrm{IF}} a^{m} x$. 
Lemma 12 Assume that, for some terms $t, u$ and substitution $\sigma$ :

1. $t \precsim \mathrm{IF} u$;

2. $m>\operatorname{depth}(u)$;

3. $\sigma(u) \precsim$ IF $a^{m} x+x$; and

4. $\sigma(t) \simeq_{\mathrm{IF}} a^{m} x$.

Then $\sigma(u) \simeq_{\mathrm{IF}} a^{m} x$.

Proof: Since $\sigma(u) \precsim_{\mathrm{IF}} a^{m} x+x$, by Lem. 11 , either $\sigma(u) \simeq_{\mathrm{IF}} a^{m} x+x$ or $\sigma(u) \simeq_{\mathrm{IF}} a^{m} x$.

Suppose, towards a contradiction, that $x$ is a summand of $\sigma(u)$. Then $u$ has a variable summand $z$ such that $x$ is a summand of $\sigma(z)$. By proviso (1) of the lemma and Lem. $1(1), t \simeq_{\mathrm{T}} u$, so clearly $z \in \operatorname{var}(t)$. Since $\operatorname{depth}(t)<m, z \in \operatorname{var}_{k}(t)$ for some $k<m$, and so $x \in \operatorname{var}_{k}(\sigma(t))$. However, since $\sigma(t) \simeq_{\mathrm{IF}} a^{m} x$, this is in contradiction with Lem. 2 .

Concluding, $x$ is not a summand of $\sigma(u)$. So by Lem. 2, $\sigma(u) \not_{\mathrm{IF}} a^{m} x+x$. Hence, $\sigma(u) \simeq_{\mathrm{IF}} a^{m} x$.

Lemma 13 Assume that, for some terms $t, u$ :

1. $E \vdash t \preccurlyeq u$;

2. $m>\max \{\operatorname{depth}(w) \mid v \preccurlyeq w \in E\} ;$

3. $u \precsim$ IF $a^{m} x+x$; and

4. $t \simeq_{\mathrm{IF}} a^{m} x$.

Then $u \simeq_{\mathrm{IF}} a^{m} x$.

Proof: By induction on the derivation of $E \vdash t \preccurlyeq u$.

- Case $E \vdash t \preccurlyeq u$ because $\sigma(v)=t$ and $\sigma(w)=u$ for some $v \preccurlyeq w \in E$ and substitution $\sigma$. The claim follows by Lem. 12 .

- Case $E \vdash t \preccurlyeq u$ because $E \vdash t \preccurlyeq v$ and $E \vdash v \preccurlyeq u$ for some $v$. By the soundness of $E$, $v \precsim_{\mathrm{IF}} u \precsim_{\mathrm{IF}} a^{m} x+x$, so by induction, $v \simeq_{\mathrm{IF}} a^{m} x$. Hence, again by induction, $u \simeq_{\mathrm{IF}} a^{m} x$.

- Case $E \vdash t \preccurlyeq u$ because $t=t^{\prime}+t^{\prime \prime}$ and $u=u^{\prime}+u^{\prime \prime}$ with $E \vdash t^{\prime} \preccurlyeq u^{\prime}$ and $E \vdash t^{\prime \prime} \preccurlyeq u^{\prime \prime}$. Without loss of generality we can assume that $t^{\prime} \neq \mathbf{0}$ and $t^{\prime \prime} \neq \mathbf{0}$. Since $t \simeq_{\mathrm{IF}} a^{m} x$, it is then not hard to see that $t^{\prime} \simeq_{\mathrm{IF}} a^{m} x$ and $t^{\prime \prime} \simeq_{\mathrm{IF}} a^{m} x$. And from $u \precsim_{\mathrm{IF}} a^{m} x+x$ and $\mathcal{I}\left(u^{\prime}\right)=\mathcal{I}\left(u^{\prime \prime}\right)=\{a\}$ (because $\left.t, t^{\prime} \neq \mathbf{0}\right)$ it follows that $u^{\prime} \precsim_{\mathrm{IF}} a^{m} x+x$ and $u^{\prime \prime} \precsim_{\mathrm{IF}} a^{m} x+x$. So by induction, $u^{\prime} \simeq_{\mathrm{IF}} a^{m} x$ and $u^{\prime \prime} \simeq_{\mathrm{IF}} a^{m} x$. Hence $u \simeq_{\mathrm{IF}} a^{m} x$.

- Case $E \vdash t \preccurlyeq u$ because $t=a t^{\prime}$ and $u=a u^{\prime}$ with $E \vdash t^{\prime} \preccurlyeq u^{\prime}$. Then $x$ is not a summand of $u$, so by Lem. 2, $u \chi_{\mathrm{IF}} a^{m} x+x$. Hence, it follows from proviso (3) of the lemma and Lem. 11 that $u \simeq \mathrm{IF} a^{m} x$.

Theorem 5 For $|A|=1$, the inequational theory of $\operatorname{BCCSP}(A)$ modulo $\precsim$ IF does not have a finite basis.

Proof: Let $E$ be a finite axiomatization over $\operatorname{BCCSP}(A)$ that is sound modulo $\precsim_{\text {IF }}$. Let $m$ be greater than the depth of any term in $E$. Since $m>1$, clearly $a^{m} x+x \neq_{\mathrm{IF}} a^{m} x$. So according to Lem. 13, $a^{m} x \preccurlyeq a^{m} x+x$ cannot be derived from $E$. And since $|A|=1$, this inequation is sound modulo $\precsim_{\mathrm{IF}}$. 


\section{7. $n$-Nested Impossible Futures}

Similar to the $n$-nested semantics and $n$-nested possible futures semantics (see, e.g., [1]), one can define $n$-nested impossible futures semantics.

Definition 4 Assume a labeled transition system. For each $n \geq 0$, the $n$-nested impossible futures preorder ${ }_{n}$ on states is defined by:

- $s_{1} \precsim 0 s_{2}$ for any states $s_{1}, s_{2}$;

- $s_{1} \precsim n+1 s_{2}$ if $s_{1} \stackrel{a_{1} \cdots a_{k}}{\rightarrow} s_{1}^{\prime}$ implies $s_{2} \stackrel{a_{1} \cdots a_{k}}{\rightarrow} s_{2}^{\prime}$ with $s_{2}^{\prime} \precsim_{n} s_{1}^{\prime}$.

We write $\simeq_{n}$ for $\precsim_{n} \cap \succsim_{n}$.

$\precsim_{n+1} \subset \simeq_{n} \subset{ }_{n}$ for $n \geq 1$. Moreover, $\precsim_{1}$ coincides with trace preorder, while $\precsim_{2}=\precsim_{\text {IF }}$. It is not hard to see that the intersection of $\precsim_{n}$ (for any $n \geq 0$ ) coincides with the intersection of $\simeq_{n}$, which in turn, coincides with bisimulation. We will argue that apart from $\precsim_{\mathrm{IF}}$, no nested impossible futures semantics allows a finite, ground-complete axiomatization.

In the proof of this result, which basically consists of a generalization of the proofs of Lem. 7, Lem. 8 and Thm. 2, we shall make use of formulas in the modal characterization of the $n$-nested impossible futures preorders. A state $s$ satisfies the modal formula $\langle a\rangle \varphi$ if there exists a transition $s \stackrel{a}{\rightarrow} s^{\prime}$ where $s^{\prime}$ satisfies the modal formula $\varphi$.

Definition 5 For $n \geq 0$, we define a set $\mathcal{L}_{n}$ of modal formulas:

$\mathcal{L}_{0}$ contains only $\top$ and $\perp$;

$\mathcal{L}_{n+1}$ is given by the $\mathrm{BNF}$

$$
\varphi::=\left\langle a_{1}\right\rangle \cdots\left\langle a_{k}\right\rangle \neg \varphi^{\prime} \quad\left(a_{1} \cdots a_{k} \in A^{*}, \varphi^{\prime} \in \mathcal{L}_{n}\right) .
$$

Lemma 14 Let $n \geq 0$. If $s_{1} \precsim_{n} s_{2}$, then $\forall \varphi \in \mathcal{L}_{n}: s_{1} \models \varphi \Rightarrow s_{2}=\varphi$.

Proof: By induction on $n$. The base case is trivial. Suppose $s_{1} \precsim_{n+1} s_{2}$, and let $s_{1} \models \varphi \in \mathcal{L}_{n+1}$, where $\varphi=\left\langle a_{1}\right\rangle \cdots\left\langle a_{k}\right\rangle \neg \varphi$ with $\varphi \in \mathcal{L}_{n}$. Then $s_{1} \stackrel{a_{1} \cdots a_{k}}{\rightarrow} s_{1}^{\prime}$ with $s_{1}^{\prime} \not \models \varphi$. Since $s_{1} \precsim_{n+1} s_{2}, s_{2} \stackrel{a_{1} \cdots a_{k}}{\rightarrow} s_{2}^{\prime}$ with $s_{2}^{\prime} \precsim{ }_{n} s_{1}^{\prime}$. By the induction hypothesis, $s_{2}^{\prime} \not \models \varphi$. Then $s_{2}^{\prime} \models \neg \varphi$, and thus $q \models \varphi$.

The operator ${ }_{-} i_{m} a^{\ell}$ adds a sequence of $\ell a$-transitions to every state at depth $m$ from which no transition is available.

Definition $6\left[1\right.$, Def. 31] For $k, \ell \geq 0$, define the operator ${ }_{-} ;_{k} a^{\ell}$ on closed terms recursively by

$$
\begin{aligned}
\left(\sum_{i=1}^{m} a_{i} p_{i}\right) ;_{k+1} a^{\ell} & =\sum_{i=1}^{m} a_{i}\left(p_{i} ;{ }_{k} a^{\ell}\right) \\
(b p+q) ;_{0} a^{\ell} & =b p+q \\
\mathbf{0} ;_{0} a^{\ell} & =a^{\ell} \mathbf{0}
\end{aligned}
$$

In the remainder of this section, we assume without loss of generality that $A=\{a\}$. This is justified because in the coming proofs we will only consider inequations $t \preccurlyeq u$ and equations $t \approx u$ where no actions $b \neq a$ occur in $t$ and $u$; and it is easy to see that any sound derivation of such an (in)equation cannot contain an occurrence of an action $b \neq a$.

For $n \geq 1$ and $m \geq 0$, we define formulae $\varphi_{n}^{m}$ :

$$
\begin{aligned}
& \varphi_{1}^{m}=\langle a\rangle^{m} \neg\langle a\rangle \top \\
& \varphi_{n+1}^{m}=\langle a\rangle \neg \varphi_{n}^{m}
\end{aligned}
$$

In other words, $\varphi_{n}^{m}=(\langle a\rangle \neg)^{n-1}\langle a\rangle^{m} \neg\langle a\rangle \top$. By induction on $n$, it is easy to see that $\varphi_{n}^{m} \in \mathcal{L}_{n+1}$.

We now formulate a slight variation of $[1$, Lem. 36]. 
Lemma 15 Let $t$ be a term with $\operatorname{depth}(t)<m$ and $\operatorname{depth}(\rho(t))<2 m+n$, for some $m, n \geq 1$. Let $\rho$ be a closed substitution with $\rho(y)=\mathbf{0}$ for each variable $y$ that occurs at multiple depths in $t$. Let $\rho^{\prime}$ be a closed substitution with $\rho^{\prime}(x)=\rho(x) ;_{m+n-1-d_{x}} a^{m+1} \mathbf{0}$ if $\rho(x) \neq \mathbf{0}$ and $x \in \operatorname{var}_{d_{x}}(t)$, and $\rho^{\prime}(x)=\mathbf{0}$ if $\rho(x)=\mathbf{0}$. Then

$$
\rho(t) \models \varphi_{n}^{m} \Leftrightarrow \rho^{\prime}(t) \models(\langle a\rangle \neg)^{n-1}\langle a\rangle^{2 m+1} \top
$$

Proof: (Sketch) By induction on $m$, we can show

$$
\rho^{\prime}(t)=\rho(t) ;_{m+n-1} a^{m+1}
$$

And by induction on $m+n$, we can show

$$
\rho(t) \models \varphi_{n}^{m} \Leftrightarrow \rho(t) ;_{m+n-1} a^{m+1} \models(\langle a\rangle \neg)^{n-1}\langle a\rangle^{2 m+1} \top
$$

(The latter proof uses that $A=\{a\}$.)

Lemma 16 Let $n \geq 1$. Assume that, for some terms $t, u$ and closed substitution $\rho$ :

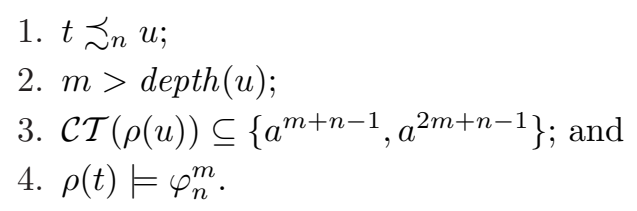

Then $\rho(u) \models \varphi_{n}^{m}$.

Proof: From provisos (2) and (3), it is not hard to see that $\rho(y)=\mathbf{0}$ for each variable $y$ that occurs at multiple depths in $u$. So by Lem. 2, the same holds for $t$. Let $\rho^{\prime}$ be defined as in Lem. 15. By proviso (4), $\rho(t)=\varphi_{n}^{m}$, so by Lem. 15, $\rho^{\prime}(t) \models(\langle a\rangle \neg)^{n-1}\langle a\rangle^{2 m+1} \top$. Note that $(\langle a\rangle \neg)^{n-1}\langle a\rangle^{2 m+1} \top \in \mathcal{L}_{n}$. By proviso $(1), \rho^{\prime}(t) \precsim_{n} \rho^{\prime}(u)$, so by Lem. 14, $\rho^{\prime}(u) \models(\langle a\rangle \neg)^{n-1}\langle a\rangle^{2 m+1} \top$. Hence, again by Lem. 15, $\rho(u) \models \varphi_{n}^{m}$.

Lemma 17 Let $n \geq 2$. Let the finite axiomatization $E$ be sound modulo $\simeq_{n}$. Assume that, for some closed terms $p, q$ :

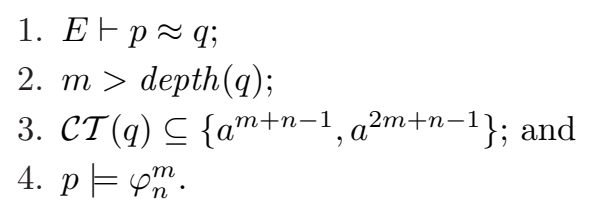

Then $q \models \varphi_{n}^{m}$.

Proof: By induction on the derivation of $E \vdash p \approx q$.

The case $\rho(t)=p$ and $\rho(u)=q$ for some $t \approx u \in E$ and closed substitution $\rho$, follows from Lem. 16 .

The other three cases $\left((1) E \vdash p \approx r\right.$ and $E \vdash r \approx q$; (2) $p=p_{1}+p_{2}$ and $q=q_{1}+q_{2}$ with $E \vdash p_{1} \approx q_{1}$ and $E \vdash p_{2} \approx q_{2} ;(3) p=a p^{\prime}$ and $q=a q^{\prime}$ with $\left.E \vdash p^{\prime} \approx q^{\prime}\right)$ can be dealt with in the same way as in the proof of Lem. 8 .

Theorem 6 Let $n \geq 2$. There is no finite, sound, ground-complete axiomatization for $\operatorname{BCCSP}(A)$ modulo $\simeq_{n}$. 
Proof: Let $E$ be a finite axiomatization that is sound modulo $\simeq_{n}$. Let $m$ be greater than the depth of any term in $E$.

For $k \geq 0$, we define closed terms $p_{k}^{m}$ and $q_{k}^{m}$ :

$$
\begin{array}{ll}
p_{0}^{m}=a^{2 m-1} \mathbf{0} & q_{0}^{m}=a^{m-1} \mathbf{0} \\
p_{k+1}^{m}=a p_{k}+a q_{k} & q_{k+1}^{m}=a p_{k}
\end{array}
$$

Clearly, $q_{k} \precsim_{k+1} p_{k}$. This induces that $p_{k}^{m} \simeq_{k} q_{k}^{m}$.

It is not hard to see that $p_{k}^{m} \models \varphi_{k}^{m}$ while $q_{k}^{m} \not \models \varphi_{k}^{m}$ (for $k \geq 1$ ). So by Lem. $17, p_{n}^{m} \approx q_{n}^{m}$ cannot be derived from $E$. Hence, $E$ is not ground-complete.

Likewise we can prove this non-finite axiomatizability result for $\precsim_{n}$ in case $n \geq 3$. The reason that the proof can be shifted from equivalences to preorders without problem, is that the key result Lem. 16 is formulated for $\precsim_{n}$. The reason that the proof does not extend to $\precsim_{2}$ is that $\precsim_{2} \beth_{\beth_{\mathrm{CT}}}$, while this inclusion is essential in the proof of Lem. 17 (see also the proof of Lem. 8). On the other hand, $\precsim_{3} \subseteq \simeq_{\mathrm{CT}}$ does hold (see Lem. 1).

Theorem 7 Let $n \geq 3$. There is no finite, sound, ground-complete axiomatization for $\operatorname{BCCSP}(A)$ modulo ${ }_{n}$.

\section{Acknowledgments}

This research was initiated by a question from Jos Baeten. We thank Bas Luttik, Marc Voorhoeve and Yanjing Wang for useful feedback. We are also indebt to two anonymous reviewers for their constructive comments. 


\section{Bibliography}

[1] L. Aceto, W. Fokkink, R. van Glabbeek and A. Ingolfsdottir. Nested semantics over finite trees are equationally hard. Inf. Comput., 191(2):203-232, 2004.

[2] L. Aceto, W. Fokkink and A. Ingolfsdottir. Ready to preorder: Get your BCCSP axiomatization for free! In Proc. CALCO'07, LNCS 4624, pp. 65-79, Springer, 2007.

[3] L. Aceto, W. Fokkink, A. Ingolfsdottir and B. Luttik. Finite equational bases in process algebra: Results and open questions. In Processes, Terms and Cycles: Steps on the Road to Infinity, LNCS 3838, pp. 338-367, Springer, 2005.

[4] T. Chen, W. Fokkink, B. Luttik and S. Nain. On finite alphabets and infinite bases. Inf. Comput. (CONCUR'06 special issue), To appear.

[5] D. de Frutos-Escrig, C. Gregorio-Rodríguez and M. Palomino. Ready to preorder: an algebraic and general proof. Submitted. Available at http://maude.sip.ucm.es/ miguelpt/.

[6] R. van Glabbeek. The linear time - branching time spectrum I. The semantics of concrete, sequential processes. In Handbook of Process Algebra, pp. 3-99, Elsevier, 2001.

[7] J.F. Groote. A new strategy for proving $\omega$-completeness with applications in process algebra. In Proc. CONCUR'90, LNCS 458, pp. 314-331, Springer, 1990.

[8] R. van Glabbeek and M. Voorhoeve. Liveness, fairness and impossible futures. In Proc. CONCUR'06, LNCS 4137, pp. 126-141, Springer, 2006.

[9] A. Rensink and W. Vogler. Fair testing. Inf. Comput., 205(2):125-198, 2007.

[10] W. Rounds and S. Brookes. Possible futures, acceptances, refusals and communicating processes. In Proc. FOCS'81, pp. 140-149. IEEE, 1981.

[11] W. Vogler. Modular construction and partial order semantics of Petri nets. LNCS 625, Springer, 1992.

[12] M. Voorhoeve and S. Mauw. Impossible futures and determinism. Inf. Proc. Lett., 80(1):51-58, 2001. 


\section{Appendix I \\ Inverted Substitutions}

Groote [7] introduced the technique of inverted substitutions to prove that an equational axiomatization is $\omega$-complete. Here we adapt his technique to make it suitable for inequational axiomatizations. Let $\mathrm{T}(\Sigma)$ and $\mathbb{T}(\Sigma)$ denote the sets of closed and open terms, respectively, over some signature $\Sigma$.

Theorem 8 Consider an inequational axiomatization $E$ over $\Sigma$. Suppose that for each inequation $t \preccurlyeq u$ of which all closed instances can be derived from $E$, there are a closed substitution $\rho$ and a mapping $R: \mathrm{T}(\Sigma) \rightarrow \mathbb{T}(\Sigma)$ such that:

(1) $E \vdash t \preccurlyeq R(\rho(t))$ and $E \vdash R(\rho(u)) \preccurlyeq u$;

(2) $E \vdash R(\sigma(v)) \preccurlyeq R(\sigma(w))$ for each $v \preccurlyeq w \in E$ and closed substitution $\sigma$; and

(3) for each function symbol $f$ (with arity $n$ ) in the signature, and all closed terms $p_{1}, \ldots, p_{n}, q_{1}, \ldots, q_{n}$ :

$$
\begin{aligned}
& E \cup\left\{p_{i} \preccurlyeq q_{i}, R\left(p_{i}\right) \preccurlyeq R\left(q_{i}\right) \mid i=1, \ldots, n\right\} \\
& R\left(f\left(p_{1}, \ldots, p_{n}\right)\right) \preccurlyeq R\left(f\left(q_{1}, \ldots, q_{n}\right)\right)
\end{aligned}
$$

Then $E$ is $\omega$-complete.

Proof: Let $t, u$ be terms such that for each closed substitution $\sigma$,

$$
E \vdash \sigma(t) \preccurlyeq \sigma(u)
$$

By assumption, there are a closed substitution $\rho$ and a mapping $R: \mathrm{T}(\Sigma) \rightarrow \mathbb{T}(\Sigma)$ such that properties $(1,2,3)$ above are satisfied. We have to prove that $E \vdash t \preccurlyeq u$. This is an immediate corollary of the following claim, for all closed terms $p, q$ :

$$
E \vdash p \preccurlyeq q \Longrightarrow E \vdash R(p) \preccurlyeq R(q)
$$

Namely, by assumption, $E \vdash \rho(t) \preccurlyeq \rho(u)$, and then the claim above implies that $E \vdash R(\rho(t)) \preccurlyeq$ $R(\rho(u))$. So by property $(1), E \vdash t \preccurlyeq u$. 
Proof of the claim. By induction on the proof of $E \vdash p \preccurlyeq q$. We have to check the four kinds of inference rules.

- $p=q$. Then $R(p)=R(q)$.

- $p \preccurlyeq q$ is an instance of some $v \preccurlyeq w \in E$ and a closed substitution $\sigma$. By property (2), $E \vdash$ $R(p) \preccurlyeq R(q)$.

- $E \vdash p \preccurlyeq q$ has been proved by $E \vdash p \preccurlyeq r$ and $E \vdash r \preccurlyeq q$, for some $r$. By induction, $E \vdash R(p) \preccurlyeq$ $R(r)$ and $E \vdash R(r) \preccurlyeq R(q)$. So $E \vdash R(p) \preccurlyeq R(q)$.

- $p=f\left(p_{1}, \ldots, p_{n}\right)$ and $q=f\left(q_{1}, \ldots, q_{n}\right)$, and $E \vdash p \preccurlyeq q$ has been proved by $E \vdash p_{i} \preccurlyeq q_{i}$ fo $i=1, \ldots, n$. By induction, $E \vdash R\left(p_{i}\right) \preccurlyeq R\left(q_{i}\right)$ for $i=1, \ldots, n$. So by property $(3), E \vdash$ $R\left(f\left(p_{1}, \ldots, p_{n}\right)\right) \preccurlyeq R\left(f\left(q_{1}, \ldots, q_{n}\right)\right)$. 